\title{
Estudo da evapotranspiração real diária utilizando imagem de satélite na bacia do rio Paracatu
}

\author{
Evaldo de Paiva Lima ${ }^{1}$ \\ Ricardo Guimarães Andrade ${ }^{2}$ \\ Gilberto Chohaku Sediyama ${ }^{1}$ \\ Rafael Coll Delgado ${ }^{1}$ \\ Anderson Soares Ferreira ${ }^{2}$ \\ Eduardo Caputi ${ }^{2}$ \\ Willian José Ferreira ${ }^{3}$ \\ ${ }^{1}$ Universidade Federal de Viçosa - UFV/DEA \\ Av. P. H. Rolfs, s/n - Campus Universitário \\ 36570-000 - Viçosa - MG, Brasil \\ lima_evaldo@yahoo.com.br \\ g.sediyama@ufv.br \\ rcdelgado@ hotmail.com \\ ${ }^{2}$ Embrapa Monitoramento por Satélite - CNPM \\ Av. Soldado Passarinho, 303 \\ 13070-115 - Campinas - SP, Brasil \\ \{ricardo, anderson, edu\}@cnpm.embrapa.br \\ ${ }^{3}$ Universidade de Taubaté - UNITAU \\ Estrada Municipal Dr. José Luiz Cembranelli, 5.000 \\ 12080-010 - Taubaté - SP, Brasil \\ will.ferreira@hotmail.com
}

\begin{abstract}
Researches related to water demands in a regional dimension on a way we can identify variations on location and time are fundamental to diagnosis and management of river basins. Satellite images have been successful in processes analysis related to soil-plant-atmosphere system. This research aimed to accomplish a preliminary study on daily real evapotranspiration on Paracatu River Basin using satellite images from Modis Sensor and Surface Energy Balance Algorithm for Land (SEBAL). To obtain instantaneous flow of energy and daily real evapotranspiration were used Modis products of reflectance containing visible and infrared areas altogether to temperature product of the surface. On September $16^{\text {th }}, 2007$, the obtained results showed enough consistence when compared to previous scientific papers, which indicate that the methodology may be used in complementary studies related to basins management.
\end{abstract}

Palavras-chave: evapotranspiration, remote sensing, river basin, evapotranspiração, sensoriamento remoto, bacia hidrográfica. 


\section{Introdução}

Nas últimas décadas a comunidade científica mundial tem enfatizado nos mais diversificados meios de comunicação a importância da preservação e/ou uso sustentável dos recursos naturais. Os cenários das mudanças climáticas globais e seus efeitos no meio ambiente, por exemplo, têm sido uma preocupação constante da humanidade. Hamada (2007) relata que o Brasil é um país que possui sua economia fortemente dependente de recursos naturais diretamente ligados ao clima na agricultura, na geração de energia hidroelétrica, entre outros setores. Os conflitos pelo uso da água podem sofrer alterações como conseqüência das mudanças do clima, por exemplo, pela alteração da demanda de água, exigindo o levantamento das questões de gerenciamento hídrico.

Neste sentido, o conhecimento das perdas por evapotranspiração (ET) e sua distribuição espacial e temporal podem ter impacto relevante na formulação de modelos relacionados à gestão dos recursos hídricos. Alguns estudos têm sido desenvolvidos objetivando a determinação da ET com base na equação do balanço de energia à superfície e técnicas de sensoriamento remoto (Bastiaanssen et al., 1998a e 1998b; Bastiaanssen, 2000; Tasumi et al., 2008). Bezerra (2004) cita que os dados obtidos através do sensoriamento remoto para o cálculo dos fluxos de energia que ocorrem na interface solo-vegetação-atmosfera utilizam-se de algoritmos para a obtenção desses elementos. Um desses é o SEBAL (Surface Energy Balance Algorithm for Land) que foi desenvolvido por Bastiaanssen et al. (1998a e 1998b). A base teórica deste algoritmo utiliza imagens obtidas pelos satélites Landsat nas bandas do visível e do infravermelho próximo. Entretanto, Allen et al. (2002) cita que a teoria é independente do tipo de satélite utilizado e o SEBAL pode ser utilizado com imagens de outros satélites desde que se utilizem os parâmetros de forma apropriada.

Diante do exposto, este trabalho teve como objetivo realizar um estudo preliminar da evapotranspiração real diária com aplicação de imagem do sensor Modis/Terra e o algoritmo SEBAL nas sub-bacias do rio Preto e ribeirão Entre Ribeiros, pertencentes à bacia do rio Paracatu.

\section{Material e Métodos}

A bacia do rio Paracatu está situada no Médio São Francisco e drena uma área de aproximadamente $45.600 \mathrm{~km}^{2}$, sendo a segunda maior sub-bacia do rio São Francisco. Conforme relatos de Rodrigues (2004) as principais sub-bacias do rio Paracatu são, pela margem direita, rio da Prata, com área de drenagem de $3.750 \mathrm{~km}^{2}$, e rio do Sono, com 5.969 $\mathrm{km}^{2}$; e, pela margem esquerda, rio Escuro, com $4.347 \mathrm{~km}^{2}$, ribeirão Entre Ribeiros, com 3.973 $\mathrm{km}^{2}$, e rio Preto, com $10.459 \mathrm{~km}^{2}$. Este estudo foca apenas as sub-bacias referentes ao rio Preto e ribeirão Entre Ribeiros, localizadas na região do Alto Paracatu, que abrange os territórios do Distrito Federal e dos Estados de Goiás e Minas Gerais (Figura 1). O uso dos recursos hídricos no alto Paracatu se destinada primordialmente ao atendimento das demandas de abastecimento urbano, dessedentação de animais e irrigação. No entanto, o consumo de água pela irrigação possui destaque considerável quando comparados com os demais (Rodrigues, 2004). As principais culturas agrícolas da região, segundo os censos agropecuários realizados pelo Instituto Brasileiro de Geografia e Estatística (IBGE) nos anos de 1970, 1975, 1980, 1985 e 1996, são: milho, soja, feijão, banana, pimentão e laranja (Latuf, 2007).

No presente trabalho foram utilizados os produtos MOD09GA (reflectância espectral de superfície, bandas 1 a 7) e o MOD11A1 (temperatura da superfície, bandas 31 e 32) para o dia 16 de setembro de 2007. Estes produtos foram obtidos gratuitamente no sítio https://wist.echo.nasa.gov/api no formato hdf e convertidos para o formato GeoTIFF com a 
utilização do programa MODIS Reprojection Tool (MRT). Também foram utilizados os dados de velocidade do vento e radiação solar, obtidos na estação meteorológica automática (A542) pertencente ao Instituto Nacional de Meteorologia (latitude $16^{\circ} 33$ ' 14 ' Sul, longitude $46^{\circ} 52^{\prime}$ 55" Oeste e $631 \mathrm{~m}$ de altitude). Informações estas, obtidas gratuitamente ao acessar o sítio http://www.inmet.gov.br/sonabra/maps/automaticas.php.

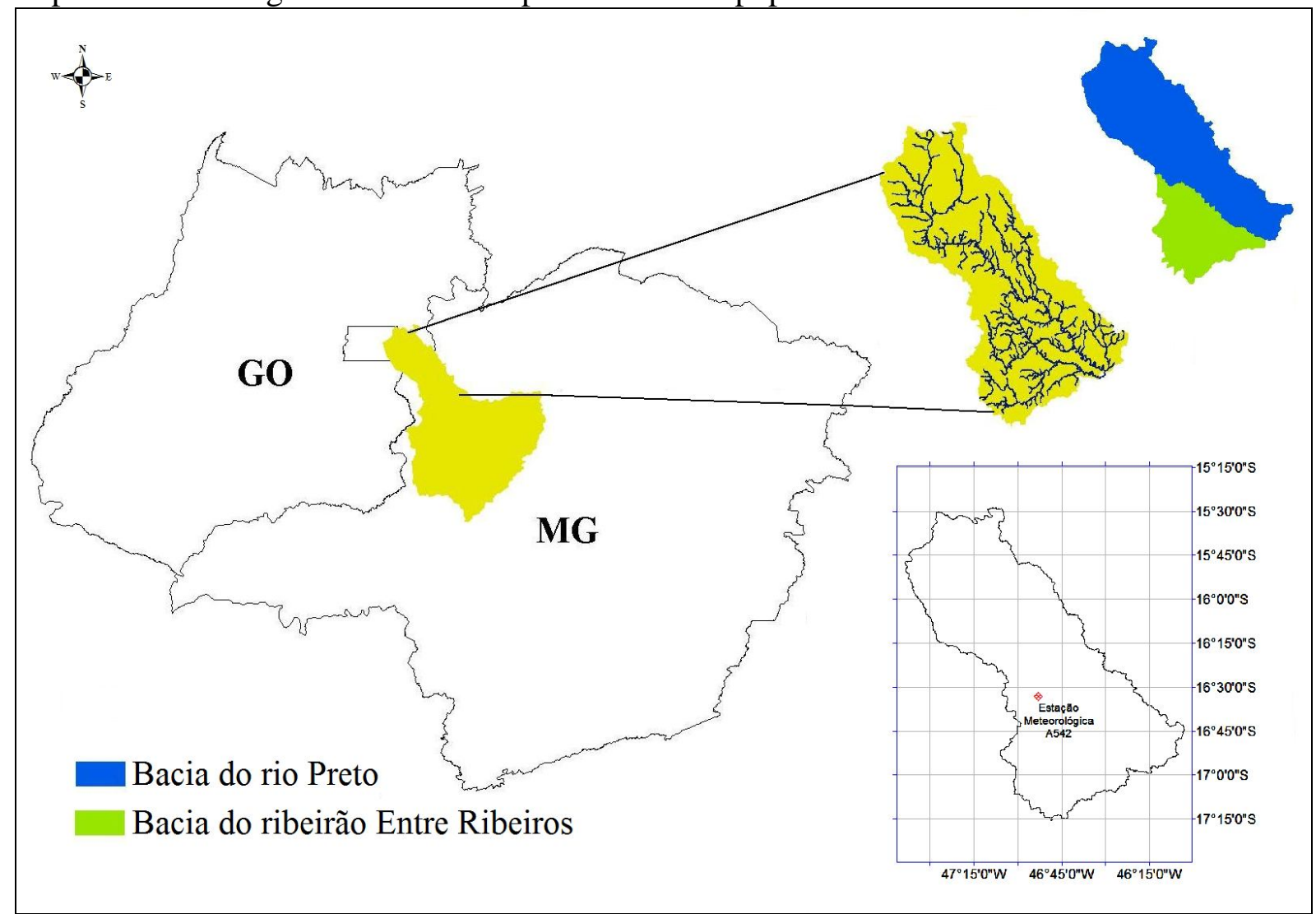

Figura 1. Localização da área de estudo.

Para estimar a evapotranspiração real diária por meio de imagem Modis e alguns dados meteorológicos foi empregado o Surface Energy Balance Algorithm for Land (SEBAL), formulado por Bastiaanssen et al. (1998a). Todas as etapas envolvidas no processo de estimativa dos componentes de energia, e conseqüentemente, na determinação da evapotranspiração real diária para a área das sub-bacias em estudo foram desenvolvidas com o auxílio da ferramenta Model Maker do programa Erdas 9.1. O saldo de radiação instantâneo (Rn) é a primeira componente do balanço de energia a ser obtida. Para tanto, aplicou-se a equação sugerida por Allen et al. (2002):

$R n=R_{s \downarrow}-\alpha R_{s \downarrow}+R_{L \downarrow}-R_{L \uparrow}-\left(1-\varepsilon_{o}\right) R_{L \downarrow}$

Em que, $R_{s \downarrow}$ é a radiação de onda curta incidente $\left(\mathrm{W} \mathrm{m}^{-2}\right), R_{L \downarrow}$ é a radiação de onda longa emitida pela atmosfera na direção da superfície $\left(\mathrm{W} \mathrm{m}^{-2}\right), R_{L \uparrow}$ é a radiação de onda longa emitida na direção da atmosfera $\left(\mathrm{W} \mathrm{m}^{-2}\right)$ e $\varepsilon_{o}$ é a emissividade da superfície (adimensional), $\alpha$ é o albedo da superfície (adimensional), obtido por meio das bandas de reflectância $\left(\alpha_{1}, \alpha_{2}, \ldots, \alpha_{7}\right)$ do produto MOD09GA juntamente com a aplicação da equação proposta por Tasumi et al. (2008):

$\alpha=0,215 \alpha_{1}+0,215 \alpha_{2}+0,242 \alpha_{3}+0,129 \alpha_{4}+0,101 \alpha_{5}+0,062 \alpha_{6}+0,036 \alpha_{7}$

Após o cálculo do Rn procedeu-se, com a aplicação da equação empírica sugerida por Bastiaanssen (2000), a estimativa do fluxo instantâneo de calor no solo (G), dado por: 
$\mathrm{G}=\left[\frac{\mathrm{T}_{\mathrm{s}}}{\alpha}\left(0,0038 \alpha+0,0074 \alpha^{2}\right)\left(1-0,98 \mathrm{NDVI}^{4}\right)\right] \mathrm{Rn}$

Em que, $\mathrm{T}_{\mathrm{s}}$ é a temperatura da superfície $\left({ }^{\circ} \mathrm{C}\right)$ relativa ao produto MOD11A1 e NDVI é o Índice de Vegetação da Diferença Normalizada (Normalized Difference Vegetation Index). Para efeito de correção dos valores do fluxo de calor no solo para corpos d'água (NDVI < 0), considerou-se $\mathrm{G}=0,3 \mathrm{Rn}$ (Silva e Bezerra, 2006).

Em seguida, obteve-se o fluxo instantâneo de calor sensível (H). Esta componente do balanço de energia expressa a taxa de calor transferido da superfície para o ar pelos processos de convecção e condução. Este fluxo é calculado utilizando a velocidade do vento (u), a resistência aerodinâmica da superfície $\left(\mathrm{r}_{\mathrm{ah}}\right)$ e a diferença da temperatura do ar $(\mathrm{dT})$ entre dois níveis acima da superfície, ou seja, nas alturas de 2,0 m e 0,1 m. No SEBAL, procedeu-se com o cálculo de dT para cada pixel por meio de uma relação linear entre dT e Ts. Esta relação é dada pelos coeficientes a e b que são obtidos pelos pixels denominados "âncoras", também chamados de "quente" e "frio". No pixel "frio" a temperatura da superfície e a do ar próxima à superfície foram consideradas iguais. Nesse caso, assumiu-se que o fluxo de calor sensível é igual a zero, e então, determinou-se o fluxo de calor latente máximo. Entretanto, no pixel "quente" assumiu-se igual a zero o fluxo de calor latente, e então, obteve-se o fluxo de calor sensível máximo. Os pixels "quente" e "frio" foram selecionados ao consultar simultaneamente as cartas do NDVI e da temperatura da superfície (Ts), ou seja, o pixel "frio" foi escolhido numa situação em que o valor do NDVI era negativo (geralmente sobre corpos d'água) com baixos valor da Ts. Entretanto, o pixel "quente" foi selecionado para condição em que a Ts era elevada e o NDVI entre 0,1 e 0,2. A Figura 2 apresenta o fluxograma das etapas utilizadas na estimativa do fluxo de calor sensível. Detalhes completos de todos os procedimentos podem ser obtidos em Allen et al. (2002).

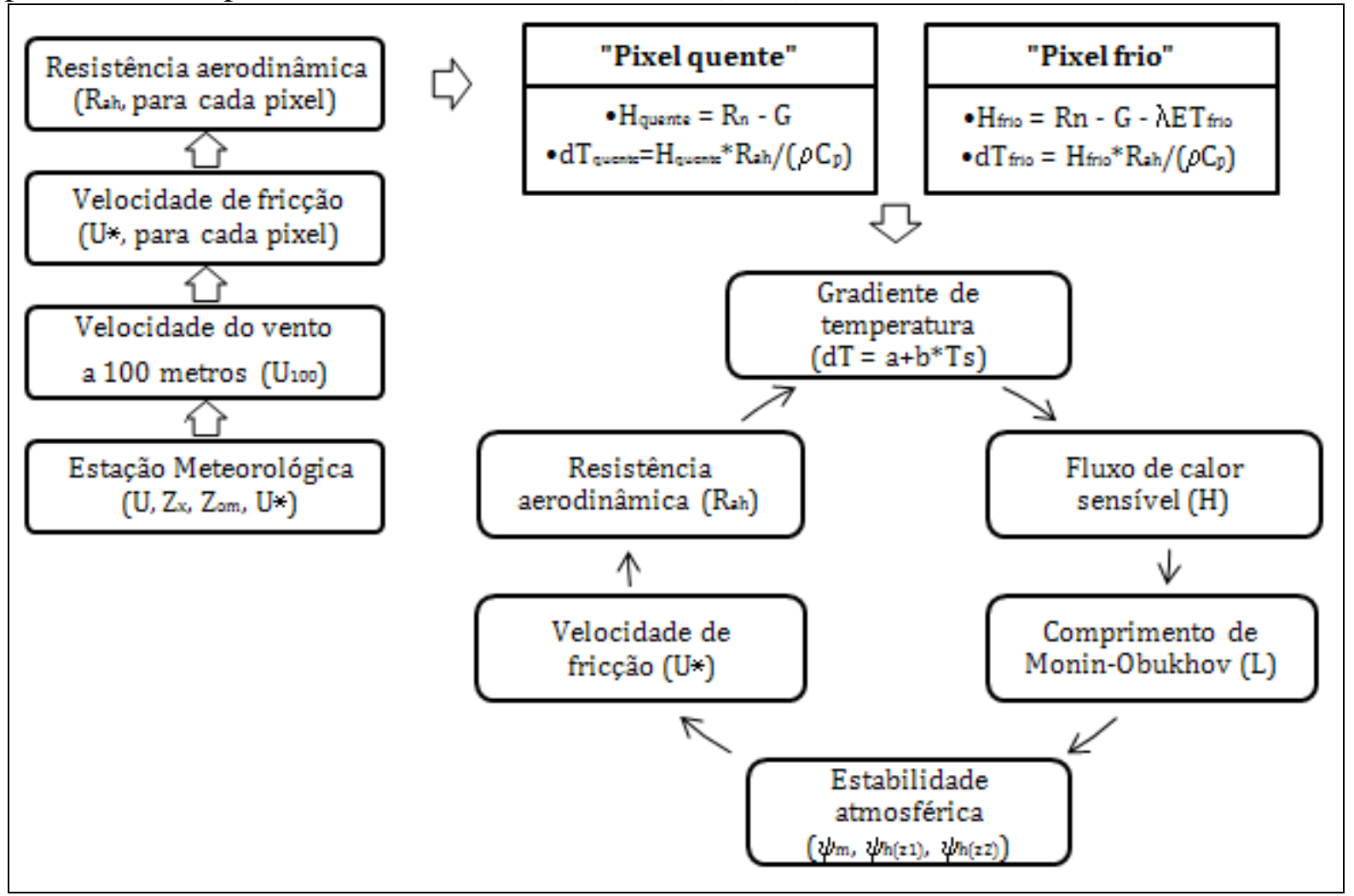

Figura 2. Fluxograma para obtenção do fluxo instantâneo de calor sensível. 
Ao estimar os valores instantâneos de Rn, G e H. Obteve-se o fluxo de calor latente instantâneo (LE) como um resíduo da equação clássica do balanço de energia (Allen et al., 2002):

$$
L E=R n-H-G
$$

Em que, LE, Rn, H e G são dados em $\mathrm{W} \mathrm{m}^{-2}$. Posteriormente, foi possível estimar a fração evaporativa (FE, adimensional) por meio da expressão sugerida por Bastiaanssen et al. (1998b):

$$
\mathrm{FE}=\frac{L E}{L E+H}=\frac{L E}{R n-G}
$$

Estudos micrometeorológicos indicam que a fração evaporativa pode ser considerada constante durante o período diurno (Crago, 1996). Assim, para a estimativa da evapotranspiração real diária $\left(\mathrm{ETr}_{\text {diária }}, \mathrm{mm} \mathrm{d}^{-1}\right)$ foi empregada a seguinte equação:

$$
E \operatorname{Tr}_{\text {diaria }}=\frac{86400 \mathrm{FE} \mathrm{Rn}}{\lambda}
$$

Em que, FE é a fração evaporativa (adimensional), $\mathrm{Rn}_{24 \mathrm{~h}}$ é o saldo de radiação médio ocorrido num período de 24 horas e $\lambda$ é calor latente de vaporização da água.

\section{Resultados e Discussão}

As Figuras 3a e 3b apresentam a distribuição espacial instantânea do albedo da superfície e saldo de radiação, respectivamente, para o dia 16 de setembro de 2007 . O albedo mínimo foi de 0,03 e o máximo foi de 0,31 , no entanto, observou-se que o albedo médio foi de 0,18 e está representado na Figura 3a pela cor verde escuro.
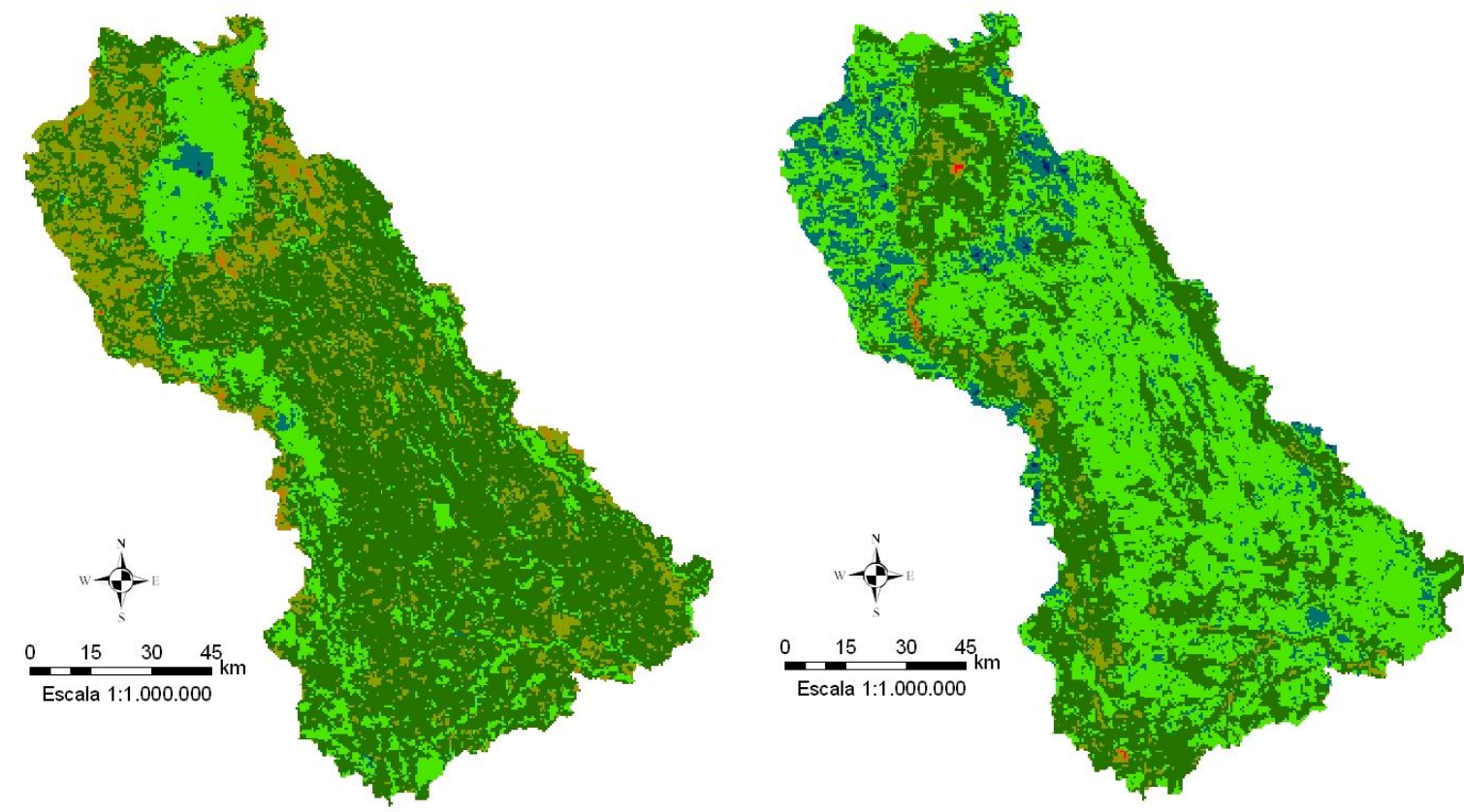

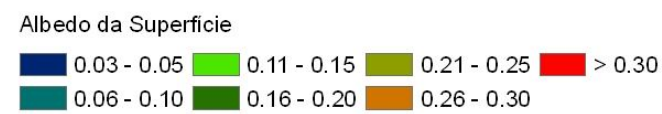

(a)

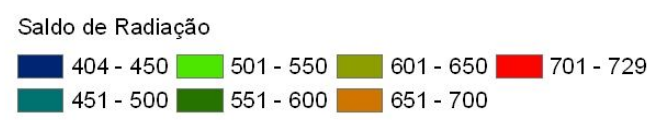

(b)

Figura 3. Distribuição espacial instantânea do albedo da superfície (a) e do saldo de radiação (b) para o dia 16 de setembro de 2007.

Para áreas de pastagem e floresta no Mato Grosso do Sul, Andrade et al. (2009a) obtiveram para o dia 05/03/2008, albedo médio de 0,23 e 0,18 para pastagem e floresta, 
respectivamente. No entanto, para o dia 08/03/2008, foram obtidos albedo médio de 0,22 e 0,17 para pastagem e floresta, respectivamente.

Com relação ao saldo de radiação ( $\mathrm{Rn})$ para o momento da passagem do satélite sobre a área da bacia, nota-se claramente que na maior extensão da área, foram encontrados valores de $\mathrm{Rn}$ variando de $500 \mathrm{~W} \mathrm{~m}^{-2}$ a $600 \mathrm{~W} \mathrm{~m}^{-2}$ (Figura 3b). Os valores mínimos e máximos de $\mathrm{Rn}$ foram de $404 \mathrm{~W} \mathrm{~m}^{-2}$ e $729 \mathrm{~W} \mathrm{~m}^{-2}$, respectivamente. Entretanto, o Rn médio foi de $542 \mathrm{~W}$ $\mathrm{m}^{-2}$. Lima et al. (2009) obtiveram para áreas irrigadas da região do Triângulo Mineiro, $\mathrm{Rn}$ variando de $307,46 \mathrm{~W} \mathrm{~m}^{-2}$ a $649,72 \mathrm{~W} \mathrm{~m}^{-2}$, com média de $529,64 \mathrm{~W} \mathrm{~m}^{-2}$.

As Figuras 4a e 4b apresentam a distribuição espacial instantânea dos fluxos de calor no solo e sensível, respectivamente, para o dia 16 de setembro de 2007. O fluxo de calor no solo (G) para o momento da passagem do satélite apresentou valores mínimo e máximo de $0 \mathrm{~W} \mathrm{~m}^{-}$ ${ }^{2}$ e $261 \mathrm{~W} \mathrm{~m}^{-2}$, respectivamente. Com média de $116 \mathrm{~W} \mathrm{~m}^{-2}$, na qual está representada na Figura 4a pela cor verde escuro, que predomina em praticamente toda a área. Silva e Bezerra (2006) ao aplicar o algoritmo SEBAL e imagem TM - Landsat 5 na região do perímetro irrigado do projeto Senador Nilo Coelho, de abrangência dos municípios de Petrolina-PE e Juazeiro-BA, encontraram para o dia 04/12/2000 e 04/10/2001 valores médios do G da ordem de $94,5 \mathrm{~W} \mathrm{~m}^{-2}$ e $112,3 \mathrm{~W} \mathrm{~m}^{-2}$, respectivamente. Em estudo realizado no Triângulo Mineiro, analisando uma série de 22 imagens TM - Landsat 5 entre os anos de 2004 e 2007, Andrade (2008) observou que o valor médio do $\mathrm{G}$ da série foi de $81,91 \mathrm{~W} \mathrm{~m}^{-2}$, no entanto, na série, os valores médios do $\mathrm{G}$ oscilaram entre $45,66 \mathrm{~W} \mathrm{~m}^{-2}$ e $269,47 \mathrm{~W} \mathrm{~m}^{-2}$ de acordo com o mês e o ano analisado.
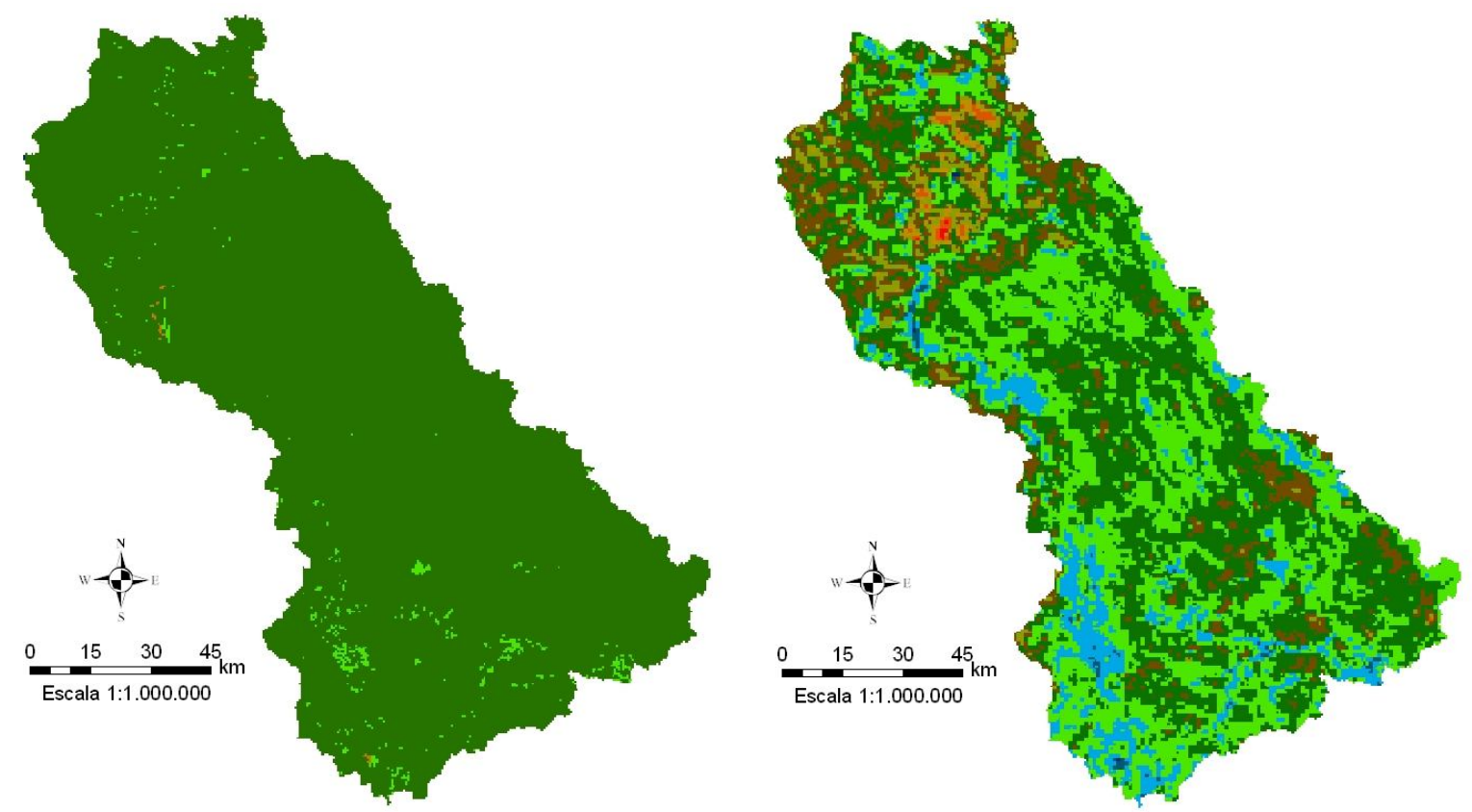

Fluxo de Calor no Solo

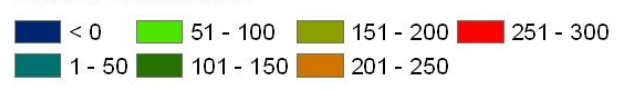

(a)

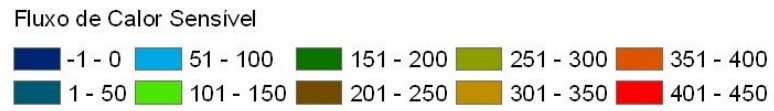

(b)

Figura 4. Distribuição espacial instantânea do fluxo de calor no solo (a) e do fluxo de calor sensível (b) para o dia 16 de setembro de 2007.

No que diz respeito ao fluxo de calor sensível $(\mathrm{H})$ instantâneo, verificou-se que os menores valores do $\mathrm{H}$ foram sobre a superfície de rios e/ou matas ciliares, representados na Figura $4 \mathbf{b}$ pelas classes de tons azuis. Entretanto, os maiores valores do $\mathrm{H}$ localizaram-se em áreas secas com baixa densidade vegetal e/ou solo descoberto (cor vermelha). $\mathrm{O}$ valor mínimo e máximo do $\mathrm{H}$ foi de $-1 \mathrm{~W} \mathrm{~m}^{-2}$ e $418 \mathrm{~W} \mathrm{~m}^{-2}$, respectivamente, com média de $159 \mathrm{~W} \mathrm{~m}^{-2}$. 
Silva e Bezerra (2006) obtiveram valores médios do $\mathrm{H}$ da ordem de $130,20 \mathrm{~W} \mathrm{~m}^{-2}$ e $158,30 \mathrm{~W}$ $\mathrm{m}^{-2}$ para imagens dos dias 04/12/2000 e 04/10/2001, respectivamente. Estes autores mostraram que os menores valores de $\mathrm{H}$ foram encontrados em superfícies de corpos d'água.

As Figuras 5a e 5b apresentam, respectivamente, a distribuição espacial do fluxo de calor latente (LE) para o momento da passagem do satélite e da evapotranspiração real diária $\left(\mathrm{ETr}_{\text {diária }}\right)$ para o dia 16 de setembro de 2007. O LE apresentou para área de estudo valores mínimos e máximos de $0 \mathrm{~W} \mathrm{~m}^{-2}$ e $616 \mathrm{~W} \mathrm{~m}^{-2}$, respectivamente, com média de $271 \mathrm{~W} \mathrm{~m}^{-2}$.

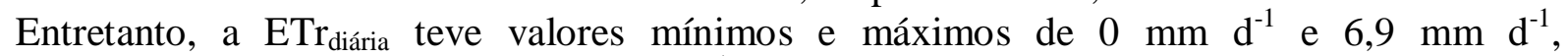
respectivamente, com média de $3,5 \mathrm{~mm} \mathrm{~d}^{-1}$. Os valores mais elevados de $\mathrm{LE}$ e $\mathrm{ETr}_{\text {diária }}$ foram observados em áreas com presença de corpos d'água (rios, lagos) e/ou nas áreas de matas ciliares. Entretanto, os menores valores de LE e ETr $_{\text {diária }}$ foram verificados nas áreas secas com pouca vegetação de cobertura e/ou com o solo totalmente exposto. Para o dia 08/03/2009 Andrade et al. (2009b) obtiveram para as áreas de pastagens da Fazenda Experimental da Embrapa Gado de Corte valores de $E T_{\text {diária }}$ oscilando de 0,51 a 2,50 $\mathrm{mm} \mathrm{d}^{-1}$. No entanto, os autores verificaram que os maiores valores de $\operatorname{ETr}_{\text {diária }}$ foram verificados em áreas ocupadas por matas ciliares (variando de 2,01 $\mathrm{mm} \mathrm{d}^{-1}$ a $3,5 \mathrm{~mm} \mathrm{~d}^{-1}$ ), com presença de corpos d'água. Entretanto, os valores mínimos foram observados em áreas com solo exposto.

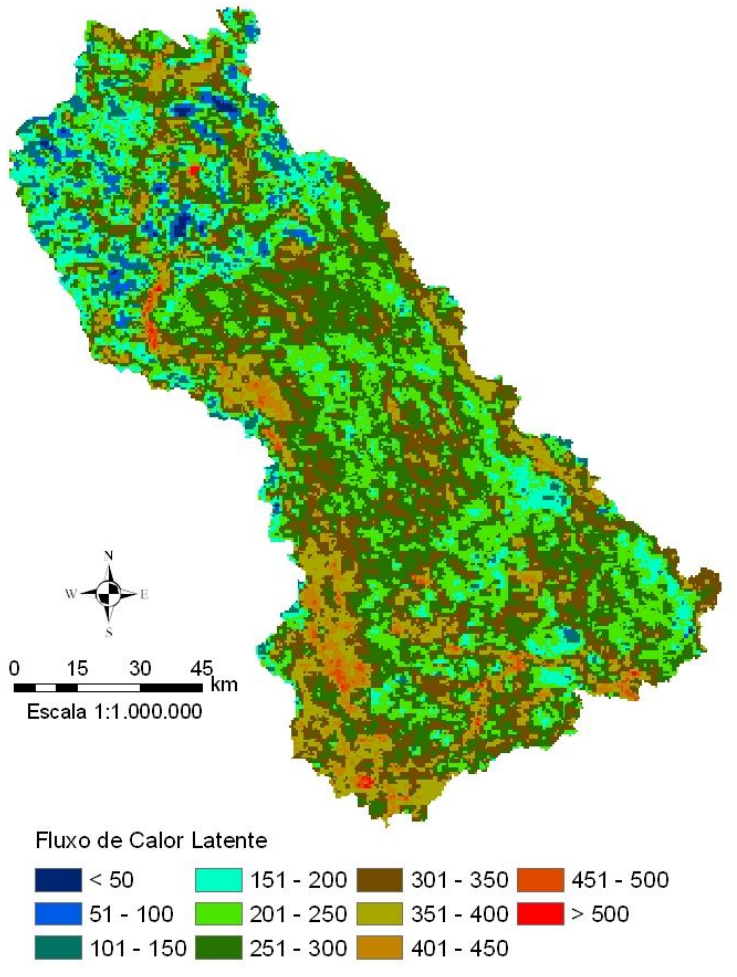

(a)

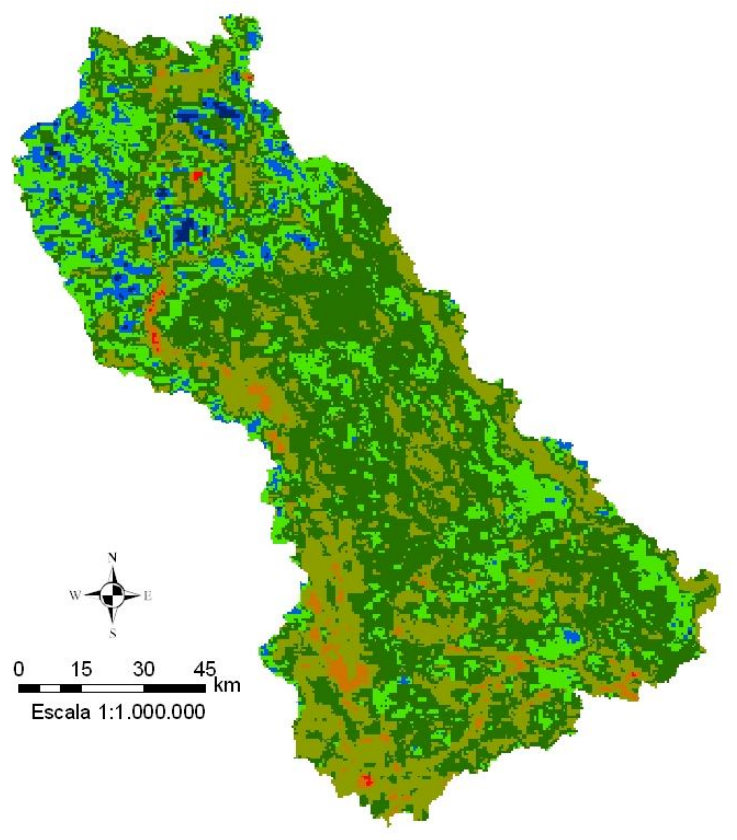

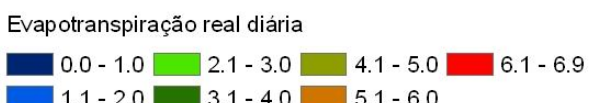

(b)

Figura 5. Distribuição espacial instantânea do fluxo de calor latente (a) e da evapotranspiração real diária (b) para o dia 16 de setembro de 2007.

\section{Conclusões}

Os resultados obtidos por meio do processo metodológico de aplicação de imagens Modis/Terra juntamente com o algoritmo SEBAL para estimativa da evapotranspiração real diária, apesar de preliminares, apresentaram consistência com dados observados na literatura. Podendo ser utilizada em estudos complementares relacionados à gestão de bacias, principalmente em regiões que possuem limitações de dados meteorológicos para uma melhor análise da variabilidade espacial e temporal da evapotranspiração. 


\section{Agradecimentos}

Ao $\mathrm{CNPq}$ pelo financiamento parcial desta pesquisa.

\section{Referências Bibliográficas}

Allen, R.; Tasumi, M.; Trezza, R.; Waters, R.; Bastiaanssen, W. Surface energy balance algorithms for land. Idaho Department of Water Resources, 2002. 97 p.

Andrade, R. G. Aplicação do algoritmo Sebal na estimativa da evapotranspiração e da biomassa acumulada da cana-de-açúcar. 2008. 135 p. Tese (Doutorado em Meteorologia Agrícola) - Universidade Federal de Viçosa, Viçosa. 2008.

Andrade, R. G.; Batistella, M.; Amaral, T. B.; Menezes, S. J. M. C. Estimativa do albedo em áreas de pastagem e floresta no Mato Grosso do Sul. In: Congresso Brasileiro de Agroinformática, 7., 2009, Viçosa. Anais... Viçosa: UFV, 2009. p. 1-5, 2009a.

Andrade, R. G.; Batistella, M.; Amaral, T. B.; Menezes, S. J. M. C. Análise preliminar da variabilidade espacial da evapotranspiração de pastagens com o uso de imagens orbitais. In: Congresso Brasileiro de Agroinformática, 7., 2009, Viçosa. Anais... Viçosa: UFV, 2009. p. 1-5, 2009 b.

Bastiaanssen, W.G.M.; Menenti, M.; Feddes, R.A.; Holtslag, A.A.M. A remote sensing surface energy balance algorithm for land (SEBAL) 1. Formulation. Journal of Hydrology, v. 212-213, p. 198-212, 1998a.

Bastiaanssen, W. G. M.; Pelgrum, H.; Wang, J.; Ma, Y.; Moreno, J. F.; Roerink, G. J.; van der Wal, T. A remote sensing surface energy balance algorithm for land (SEBAL): 2. Validation. Journal of Hydrology, v. 212213 , p. 213-229, 1998 b.

Bastiaanssen, W. G. M. SEBAL-based sensible and latent heat fluxes in the irrigated Gediz Basin, Turkey. Journal of Hydrology, v. 229, p. 87-100, 2000.

Bezerra, M. V. C. Balanço de energia em áreas irrigadas utilizando técnicas de sensoriamento remoto. 2004. 108 p. Dissertação (Mestrado em Meteorologia) - Universidade Federal de Campina Grande, Campina Grande. 2004.

Crago, R. D. Conservation and variability of the evaporative fraction during the daytime. Journal of Hidrology, v. 180, p. 173-194, 1996.

Hamada, E. Mudanças climáticas globais e recursos hídricos com enfoque para as bacias hidrográficas. In: Simpósio Internacional em Manejo de Microbacias - Sustentabilidade Ambiental da Água e Aquecimento Global, 2., 2007, Botucatu. Anais... Botucatu: UNESP/FCA, 2007. p. 1-11. Disponível em: $<$ http://www.fca.unesp.br/microbacias/artigos/Efeito_mudancas_climaticas\%20Emilia\%20Hamada.pdf $>$. Acesso em: 03 out. 2009.

Latuf, M. O. Mudanças no uso do solo e comportamento hidrológico nas bacias do rio Preto e ribeirão Entre Ribeiros. 2007. 103 p. Dissertação (Mestrado em Engenharia Agrícola) - Universidade Federal de Viçosa, Viçosa. 2007.

Lima, E. P.; Sediyama, G. C.; Gleriani, J. M.; Soares, V. P.; Delgado, R. C.; Andrade, R. G. Estimativa do balanço de radiação em áreas irrigadas utilizando imagem do Landsat 5 - TM. In: Simpósio Brasileiro de Sensoriamento Remoto, 14., 2009, Natal. Anais... São José dos Campos: INPE, 2009. p. 255-262. Disponível em: <http://marte.dpi.inpe.br/col/dpi.inpe.br/sbsr@80/2008/11.17.20.49/doc/255-262.pdf>. Acesso em: 03 out. 2009.

Rodriguez, R. D. G. Metodologia para a estimativa das demandas e disponibilidades hídricas: Estudo de caso da bacia do Paracatu. 2004. 94 p. Dissertação (Mestrado em Engenharia Agrícola) - Universidade Federal de Viçosa, Viçosa. 2004.

Silva, B. B.; Bezerra, M. V. C. Determinação dos fluxos de calor sensível e latente na superfície utilizando imagens TM - Landsat 5. Revista Brasileira de Agrometeorologia, v. 14, n. 2, p. 174-186, 2006.

Tasumi, M.; Allen, R. G.; Trezza, R. At-surface reflectance and albedo from satellite for operational calculation of Land surface energy balance. Journal of Hydrologic Engineering, v. 13, n. 2, p. 51-63, 2008. 